\title{
Spectra and Light Curves of Gamma-Ray Burst Afterglows
}

\section{Citation}

Sari, Re 'em, Tsvi Piran, and Ramesh Narayan. 1998. "Spectra and Light Curves of Gamma-Ray Burst Afterglows." The Astrophysical Journal 497 (1): L17-20. https://doi.org/10.1086/311269.

\section{Permanent link}

http://nrs.harvard.edu/urn-3:HUL.InstRepos:41384914

\section{Terms of Use}

This article was downloaded from Harvard University's DASH repository, and is made available under the terms and conditions applicable to Other Posted Material, as set forth at http:// nrs.harvard.edu/urn-3:HUL.InstRepos:dash.current.terms-of-use\#LAA

\section{Share Your Story}

The Harvard community has made this article openly available.

Please share how this access benefits you. Submit a story.

\section{Accessibility}




\title{
Spectra and Light Curves of Gamma-Ray Burst Afterglows
}

\author{
Re'em Sari, Tsvi Piran \\ Racah Institute, Hebrew University, Jerusalem 91904, Israel \\ and \\ Ramesh Narayan \\ Harvard-Smithsonian Center for Astrophysics, 60 Garden Street, Cambridge MA 02138, U.S.A.
}

\begin{abstract}
The recently discovered GRB afterglow is believed to be described reasonably well by synchrotron emission from a slowing down relativistic shell that collides with an external medium. To compare theoretical models with afterglow observations we calculate here the broad band spectrum and corresponding light curve of synchrotron radiation from a power-law distribution of electrons in an expanding relativistic shock. Both the spectrum and the light curve consist of several power-law segments. The light curve is constructed under two limiting models for the hydrodynamical evolution of the shock: fully adiabatic and fully radiative. We compare the results with observations of $\gamma$-ray burst afterglows.
\end{abstract}

\section{Introduction}

Delayed emission in X-ray, optical and radio wavelengths has been recently seen in a few $\gamma$-ray bursts (Costa et al. 1997, Groot et al. 1997, Frail et. al. 1997). This so-called "afterglow" is described reasonably well as synchrotron emission from accelerated electrons when a spherical relativistic shell collides with an external medium (Waxman 1997a,b, Wijers, Rees \& Mészáros 1997, Katz \& Piran 1997). Previous analyses have described the spectrum and light curve only over a limited range of frequency and time. In this Letter we discuss the spectrum over a wide range of frequency and derive the shape of the light curve from very early to late times. We focus on the optical and X-ray emission, where synchrotron self absorption is not important, and we assume that the shell is ultra relativistic. We allow for both adiabatic and radiative hydrodynamical evolution.

Consider a relativistic shock propagating through a uniform cold medium with particle density $n$. Behind the shock, the particle density and the energy density are given by $4 \gamma n$ and $4 \gamma^{2} n m_{p} c^{2}$, respectively, where $\gamma$ is the Lorentz factor of the shocked fluid (Blandford \& McKee 1976). We assume that electrons are accelerated in the shock to a power law distribution of Lorentz factor $\gamma_{e}$, with a minimum Lorentz factor $\gamma_{m}: N\left(\gamma_{e}\right) d \gamma_{e} \propto \gamma_{e}^{-p} d \gamma_{e}, \gamma_{e} \geq \gamma_{m}$. To keep the energy of the electrons finite we take $p>2$. We assume that a constant fraction $\epsilon_{e}$ of the shock energy goes into the electrons. Then

$$
\gamma_{m}=\epsilon_{e}\left(\frac{p-2}{p-1}\right) \frac{m_{p}}{m_{e}} \gamma \cong 610 \epsilon_{e} \gamma,
$$

where the coefficient on the right corresponds to the standard choice, $p=2.5$ (Sari, Narayan \& Piran 1996). We also assume that the magnetic energy density behind the shock is a constant fraction $\epsilon_{B}$ of the shock energy. This gives a magnetic field strength

$$
B=\left(32 \pi m_{p} \epsilon_{B} n\right)^{1 / 2} \gamma c .
$$


In this Letter we consider only synchrotron emission, and neglect inverse Compton scattering (which can be important when $\epsilon_{B}>\epsilon_{e}$, Sari et al. 1996).

\section{Synchrotron Spectrum of a Relativistic Shock}

A relativistic electron with Lorentz factor $\gamma_{e} \gg 1$ in a magnetic field $B$ emits synchrotron radiation. The radiation power and the characteristic frequency are given by (Rybicki \& Lightman 1976)

$$
\begin{gathered}
P\left(\gamma_{e}\right)=\frac{4}{3} \sigma_{T} c \gamma^{2} \gamma_{e}^{2} \frac{B^{2}}{8 \pi}, \\
\nu\left(\gamma_{e}\right)=\gamma \gamma_{e}^{2} \frac{q_{e} B}{2 \pi m_{e} c},
\end{gathered}
$$

where the factors of $\gamma^{2}$ and $\gamma$ are introduced to transform the results from the frame of the shocked fluid to the frame of the observer. The spectral power, $P_{\nu}$ (power per unit frequency, $\operatorname{erg} \mathrm{Hz}^{-1} \mathrm{~s}^{-1}$ ), varies as $\nu^{1 / 3}$ for $\nu<\nu\left(\gamma_{e}\right)$, and cuts off exponentially for $\nu>\nu\left(\gamma_{e}\right)$ (Rybicki \& Lightman 1976). The peak power occurs at $\nu\left(\gamma_{e}\right)$, where it has the approximate value

$$
P_{\nu, \max } \approx \frac{P\left(\gamma_{e}\right)}{\nu\left(\gamma_{e}\right)}=\frac{m_{e} c^{2} \sigma_{T}}{3 q_{e}} \gamma B
$$

Note that $P_{\nu, \max }$ does not depend on $\gamma_{e}$, whereas the position of the peak does.

The above description of $P_{\nu}$ describes the emitted spectrum when the electron does not lose a significant fraction of its energy to radiation. This requires $\gamma_{e}$ to be less than a critical value $\gamma_{c}$, above which cooling by synchrotron radiation is significant. The critical electron Lorentz factor $\gamma_{c}$ is given by the condition $\gamma \gamma_{c} m_{e} c^{2}=P\left(\gamma_{c}\right) t$

$$
\gamma_{c}=\frac{6 \pi m_{e} c}{\sigma_{T} \gamma B^{2} t}=\frac{3 m_{e}}{16 \epsilon_{B} \sigma_{T} m_{p} c} \frac{1}{t \gamma^{3} n},
$$

where $t$ refers to time in the frame of the observer.

Consider now an electron with an initial Lorentz factor $\gamma_{e}>\gamma_{c}$. This electron cools down to $\gamma_{c}$ in the time $t$. As it cools, the frequency of the synchrotron emission varies as $\nu \propto \gamma_{e}^{2}$ while the electron energy varies as $\gamma_{e}$. It then follows that the spectral power varies as $\nu^{-1 / 2}$ over the frequency range $\nu_{c}<\nu<\nu\left(\gamma_{e}\right)$, where we have defined $\nu_{c} \equiv \nu\left(\gamma_{c}\right)$. The net spectrum of radiation from such an electron then consists of three segments, a low energy tail for $\nu<\nu_{c}$ where $P_{\nu}$ goes as $\nu^{1 / 3}$, a power-law segment between $\nu_{c}$ and $\nu\left(\gamma_{e}\right)$ where $P_{\nu} \sim \nu^{-1 / 2}$, and an exponential cutoff for $\nu>\nu\left(\gamma_{e}\right)$. The maximum emissivity occurs at $\nu_{c}$ and is given by $P_{\nu, \max }$.

As described in the previous section, we are interested in a power-law distribution of electrons. To calculate the net spectrum due to all the electrons we need to integrate over $\gamma_{e}$. There are now two different cases, depending on whether $\gamma_{m}>\gamma_{c}$ or $\gamma_{m}<\gamma_{c}$.

Let the total number of electrons be $N_{e}$. When $\gamma_{m}>\gamma_{c}$, all the electrons cool down roughly to $\gamma_{c}$ and the flux at $\nu_{c}$ is approximately $N_{e} P_{\nu, \max }$. We call this the case of fast cooling. The flux at the observer, $F_{\nu}$, is given by

$$
F_{\nu}= \begin{cases}\left(\nu / \nu_{c}\right)^{1 / 3} F_{\nu, \max }, & \nu_{c}>\nu \\ \left(\nu / \nu_{c}\right)^{-1 / 2} F_{\nu, \max }, & \nu_{m}>\nu>\nu_{c} \\ \left(\nu_{m} / \nu_{c}\right)^{-1 / 2}\left(\nu / \nu_{m}\right)^{-p / 2} F_{\nu, \max }, & \nu>\nu_{m}\end{cases}
$$


where $\nu_{m} \equiv \nu\left(\gamma_{m}\right)$ and $F_{\nu, \max } \equiv N_{e} P_{\nu, \max } / 4 \pi D^{2}$ is the observed peak flux at distance $D$ from the source.

When $\gamma_{c}>\gamma_{m}$, only those electrons with $\gamma_{e}>\gamma_{c}$ can cool. We call this slow cooling, because the electrons with $\gamma_{e} \sim \gamma_{m}$, which form the bulk of the population, do not cool within a time $t$. Integrating over the electron distribution gives

$$
F_{\nu}= \begin{cases}\left(\nu / \nu_{m}\right)^{1 / 3} F_{\nu, \max }, & \nu_{m}>\nu \\ \left(\nu / \nu_{m}\right)^{-(p-1) / 2} F_{\nu, \max }, & \nu_{c}>\nu>\nu_{m} \\ \left(\nu_{c} / \nu_{m}\right)^{-(p-1) / 2}\left(\nu / \nu_{c}\right)^{-p / 2} F_{\nu, \max }, & \nu>\nu_{c}\end{cases}
$$

Typical spectra corresponding to fast and slow cooling are shown in Figures 1a and 1b. In addition to the various power-law regimes described above, self-absorption causes a steep cutoff of the spectrum, either as $\nu^{2}$ or $\nu^{5 / 2}$, at low frequencies (Katz 1994, Waxman 1997b, Katz and Piran 1997). For completeness, we show this regime in Figure 1, but we shall ignore it for the rest of this Letter since self-absorption does not affect either the optical or X-ray radiation in which we are interested.

\section{Hydrodynamical Evolution and Light Curves}

The instantaneous spectra described in the previous section do not depend on the hydrodynamical evolution of the shock. The only assumption made there is that the shock properties are fairly constant over a time scale comparable to the observation time $t$. The light curves at a given frequency, however, depend on the temporal evolution of various quantities, such as the break frequencies $\nu_{m}$ and $\nu_{c}$ and the peak power $N_{e} P_{\nu, \max }$. These depend, in turn, on how $\gamma$ and $N_{e}$ scale as a function of $t$.

We limit the discussion here to the case of a spherical shock of radius $R(t)$ propagating into a constant surrounding density $n$. Clearly, the total number of swept-up electrons in the post-shock fluid is $N_{e}=4 \pi R^{3} n / 3$. We consider two extreme limits for the hydrodynamical evolution of the shock: either fully radiative or fully adiabatic. The radiative solution assumes that all the internal energy created in the shock is radiated. This requires two conditions to be satisfied: (1) the fraction of the energy going into the electrons must be large, i.e. $\epsilon_{e} \rightarrow 1$, and (2) we must be in the regime of fast cooling, $\gamma_{c}<\gamma_{m}$. If either of these conditions is not satisfied, i.e. if $\epsilon_{e} \ll 1$ or $\gamma_{c} \gg \gamma_{m}$, then we have adiabatic evolution.

In the adiabatic case, the energy $E$ of the spherical shock is constant and is given by $E=16 \pi \gamma^{2} R^{3} n m_{p} c^{2} / 17$ (Blandford \& McKee 1976, Sari 1997). In the radiative case, the energy varies as $E \propto \gamma$, where $\gamma \cong(R / L)^{-3}$. Here $L=\left[17 M /\left(16 \pi m_{p} n\right)\right]^{1 / 3}$ (Blandford \& McKee 1976, Vietri 1996, Katz \& Piran 1997) is the radius at which the mass swept up from the external medium equals the initial mass $M$ of the ejecta (We used $17 / 16$ instead of $3 / 4$ in order to be compatible with the adiabatic expression and to enable a smooth transition between the two); we write $M$ in terms of the initial energy of the explosion via $M=E / \gamma_{0} c^{2}$, where $\gamma_{0}$ is the initial Lorentz factor of the ejecta.

In both the adiabatic and radiative cases, there is a simple relation connecting the radius of the shock $R$, the fluid Lorentz factor $\gamma$, and the observed time $t: t=R / c_{t} \gamma^{2} c$, where the numerical value of $c_{t}$ lies between $\sim 3$ and $\sim 7$ depending on the details of the hydrodynamical evolution and the spectrum (Sari 1997a, Waxman 1997c, Sari 1997b, Panaitescu \& Mészáros 1997). For simplicity we use $t \cong R / 4 \gamma^{2} c$ for all cases. We then have the following hydrodynamical evolution equations,

$$
R(t) \cong \begin{cases}\left(17 E t / 4 \pi m_{p} n c\right)^{1 / 4}, & \text { adiabatic } \\ (4 c t / L)^{1 / 7} L, & \text { radiative }\end{cases}
$$



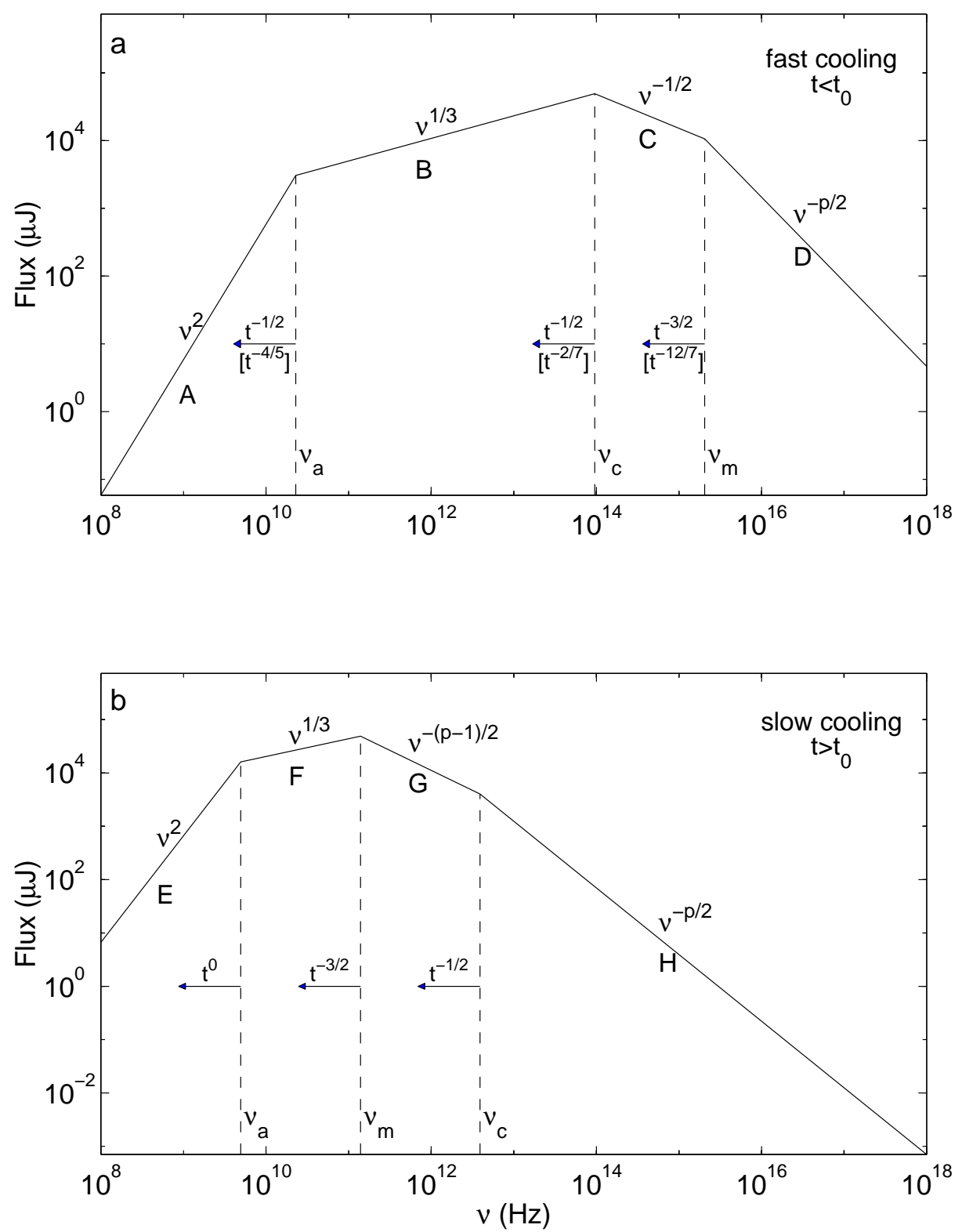

Fig. 1. - Synchrotron spectrum of a relativistic shock with a power-law distribution of electrons. (a) The case of fast cooling, which is expected at early times $\left(t<t_{0}\right)$ in a $\gamma$-ray burst afterglow. The spectrum consists of four segments, identified as A, B, C, D. Self-absorption is important below $\nu_{a}$. The frequencies, $\nu_{m}, \nu_{c}$, $\nu_{a}$, decrease with time as indicated; the scalings above the arrows correspond to an adiabatic evolution, and the scalings below, in square brackets, to a fully radiative evolution. (b) The case of slow cooling, which is expected at late times $\left(t>t_{0}\right)$. The evolution is always adiabatic. The four segments are identified as $\mathrm{E}, \mathrm{F}$, G, H. 


$$
\gamma(t) \cong \begin{cases}\left(17 E / 1024 \pi n m_{p} c^{5} t^{3}\right)^{1 / 8}, & \text { adiabatic, } \\ (4 c t / L)^{-3 / 7}, & \text { radiative }\end{cases}
$$

Using these scalings and the results of the previous section, we can calculate the variation with time of all the relevant quantities. For the adiabatic case, we find

$$
\begin{aligned}
\nu_{c} & =2.7 \times 10^{12} \epsilon_{B}^{-3 / 2} E_{52}^{-1 / 2} n_{1}^{-1} t_{d}^{-1 / 2} \mathrm{~Hz}, \\
\nu_{m} & =5.7 \times 10^{14} \epsilon_{B}^{1 / 2} \epsilon_{e}^{2} E_{52}^{1 / 2} t_{d}^{-3 / 2} \mathrm{~Hz}, \\
F_{\nu, \max } & =1.1 \times 10^{5} \epsilon_{B}^{1 / 2} E_{52} n_{1}^{1 / 2} D_{28}^{-2} \mu \mathrm{J},
\end{aligned}
$$

where $t_{d}$ is the time in days, $E_{52}=E / 10^{52} \mathrm{ergs}, n_{1}$ is $n$ in units of $\mathrm{cm}^{-3}$ and $D_{28}=D / 10^{28} \mathrm{~cm}$. In the case of a fully radiative evolution, the results are

$$
\begin{aligned}
\nu_{c} & =1.3 \times 10^{13} \epsilon_{B}^{-3 / 2} E_{52}^{-4 / 7} \gamma_{2}^{4 / 7} n_{1}^{-13 / 14} t_{d}^{-2 / 7} \mathrm{~Hz}, \\
\nu_{m} & =1.2 \times 10^{14} \epsilon_{B}^{1 / 2} \epsilon_{e}^{2} E_{52}^{4 / 7} \gamma_{2}^{-4 / 7} n_{1}^{-1 / 14} t_{d}^{-12 / 7} \mathrm{~Hz}, \\
F_{\nu, \max } & =4.5 \times 10^{3} \epsilon_{B}^{1 / 2} E_{52}^{8 / 7} \gamma_{2}^{-8 / 7} n_{1}^{5 / 14} D_{28}^{-2} t_{d}^{-3 / 7} \mu \mathrm{J},
\end{aligned}
$$

where we have scaled the initial Lorentz factor of the ejecta by a factor of 100: $\gamma_{2} \equiv \gamma_{0} / 100$.

The spectra presented in Figure 1 show the positions of $\nu_{c}$ and $\nu_{m}$ for typical parameters. Note that, in both the adiabatic and radiative cases, $\nu_{c}$ decreases more slowly with time than $\nu_{m}$. Therefore, at sufficiently early times we have $\nu_{c}<\nu_{m}$, i.e. fast cooling, while at late times we have $\nu_{c}>\nu_{m}$, i.e., slow cooling. The transition between the two occurs when $\nu_{c}=\nu_{m}$. This corresponds to the time

$$
t_{0}= \begin{cases}210 \epsilon_{B}^{2} \epsilon_{e}^{2} E_{52} n_{1} \text { days, } & \text { adiabatic, } \\ 4.6 \epsilon_{B}^{7 / 5} \epsilon_{e}^{7 / 5} E_{52}^{4 / 5} \gamma_{2}^{-4 / 5} n_{1}^{3 / 5} \text { days, } & \text { radiative }\end{cases}
$$

At $t=t_{0}$, the spectrum changes from fast cooling (Fig. 1a) to slow cooling (Fig. 1b). In addition, if $\epsilon_{e} \rightarrow 1$, the hydrodynamical evolution changes from radiative to adiabatic. However, if $\epsilon_{e} \ll 1$, the evolution remains adiabatic throughout.

Once we know how the break frequencies, $\nu_{c}, \nu_{m}$, and the peak flux $F_{\nu, \max }$ vary with time, we can calculate the light curve. Consider a fixed frequency $\nu=10^{15} \nu_{15} \mathrm{~Hz}$. From the first two equations in (11) and (12) we see that there are two critical times, $t_{c}$ and $t_{m}$, when the break frequencies, $\nu_{c}$ and $\nu_{m}$, cross the observed frequency $\nu$ :

$$
\begin{aligned}
t_{c} & = \begin{cases}7.3 \times 10^{-6} \epsilon_{B}^{-3} E_{52}^{-1} n_{1}^{-2} \nu_{15}^{-2} \text { days, } & \text { adiabatic, } \\
2.7 \times 10^{-7} \epsilon_{B}^{-21 / 4} E_{52}^{-2} \gamma_{2}^{2} n_{1}^{-13 / 4} \nu_{15}^{-7 / 2} \text { days, } & \text { radiative, }\end{cases} \\
t_{m} & = \begin{cases}0.69 \epsilon_{B}^{1 / 3} \epsilon_{e}^{4 / 3} E_{52}^{1 / 3} \nu_{15}^{-2 / 3} \text { days, } & \text { adiabatic, } \\
0.29 \epsilon_{B}^{7 / 24} \epsilon_{e}^{7 / 6} E_{52}^{1 / 3} \gamma_{2}^{-1 / 3} \nu_{15}^{-7 / 12} n_{1}^{-1 / 24} \text { days, } & \text { radiative }\end{cases}
\end{aligned}
$$

It is easily seen that there are only two possible orderings of the three critical times, $t_{c}, t_{m}, t_{0}$, namely $t_{0}>t_{m}>t_{c}$ and $t_{0}<t_{m}<t_{c}$. Let us define the critical frequency, $\nu_{0}=\nu_{c}\left(t_{0}\right)=\nu_{m}\left(t_{0}\right)$,

$$
\nu_{0}= \begin{cases}1.8 \times 10^{11} \epsilon_{B}^{-5 / 2} \epsilon_{e}^{-1} E_{52}^{-1} n_{1}^{-3 / 2} \mathrm{~Hz}, & \text { adiabatic } \\ 8.5 \times 10^{12} \epsilon_{B}^{-19 / 10} \epsilon_{e}^{-2 / 5} E_{52}^{-4 / 5} \gamma_{2}^{4 / 5} n_{1}^{-11 / 10} \mathrm{~Hz}, & \text { radiative }\end{cases}
$$

When $\nu>\nu_{0}$, the ordering $t_{0}>t_{m}>t_{c}$ applies and we refer to the corresponding light curve as the high frequency light curve. Similarly, when $\nu<\nu_{0}$, we have $t_{0}<t_{m}<t_{c}$, and we obtain the low frequency light curve. 

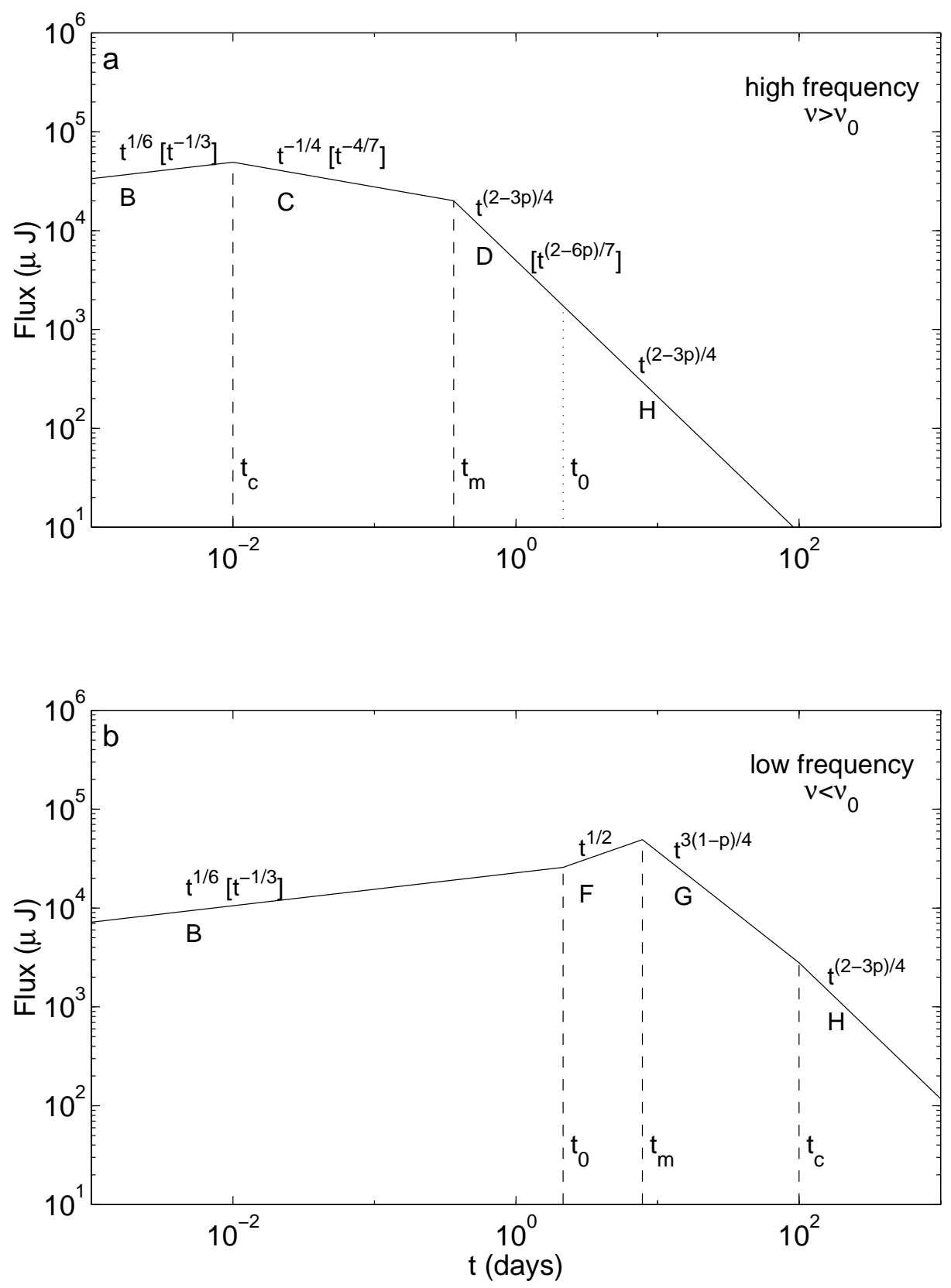

Fig. 2.- Light curve due to synchrotron radiation from a spherical relativistic shock, ignoring the effect of self-absorption. (a) The high frequency case $\left(\nu>\nu_{0}\right)$. The light curve has four segments, separated by the critical times, $t_{c}, t_{m}, t_{0}$. The labels, B, C, D, H, indicate the correspondence with spectral segments in Fig. 1. The observed flux varies with time as indicated; the scalings within square brackets are for radiative evolution (which is restricted to $t<t_{0}$ ) and the other scalings are for adiabatic evolution. (b) The low frequency case $\left(\nu<\nu_{0}\right)$. 
Figure 2a shows a typical high frequency light curve. At early times the electrons cool fast and $\nu$ is less than both $\nu_{m}$ and $\nu_{c}$. Ignoring self absorption, the situation corresponds to segment B in Figure 1, and the flux varies as $F_{\nu} \sim F_{\nu, \max }\left(\nu / \nu_{c}\right)^{1 / 3}$. If the evolution is adiabatic, $F_{\nu, \max }$ is constant, and $F_{\nu} \sim t^{1 / 6}$. In the radiative case, $F_{\nu, \max } \sim t_{d}^{-3 / 7}$ and $F_{\nu} \sim t^{-1 / 3}$. The scalings in the other segments, which correspond to C, $\mathrm{D}, \mathrm{H}$ in Fig. 1, can be derived in a similar fashion and are shown in Fig. 2a.

Figure $2 \mathrm{~b}$ shows the low frequency light curve, corresponding to $\nu<\nu_{0}$. In this case, there are four phases in the light curve, corresponding to segments B, F, G and H. The time dependences of the flux are indicated on the plot for both the adiabatic and radiative cases.

We conclude with two comments. First, during radiative evolution the energy in the shock decreases with time, the energy to be substituted in the radiative scalings is the initial energy. When a radiative shock switches to adiabatic evolution at time $t=t_{0}$, it is necessary to use the reduced energy to calculate the subsequent adiabatic evolution. The final energy $E_{f, 52}$ which one should use in the adiabatic regime is related to the initial $E_{i, 52}$ of the fireball by

$$
E_{f, 52}=0.022 \epsilon_{B}^{-3 / 5} \epsilon_{e}^{-3 / 5} E_{i, 52}^{4 / 5} \gamma_{2}^{-4 / 5} n_{1}^{-2 / 5} .
$$

Second, if during the phase of fast cooling $\left(t<t_{0}\right) \epsilon_{e}$ is somewhat less than unity, then only a fraction of the shock energy is lost to radiation. The scalings will be intermediate between the two limits of fully radiative and fully adiabatic discussed here.

\section{Discussion}

The main results of this Letter are summarized in Fig. 1 and Fig. 2, along with the scalings given in equations (11)-(17).

It is well-known that the flux at the peak of the synchrotron spectrum is independent of time in the slow cooling limit for adiabatic hydrodynamic evolution (Mészáros \& Rees 1996). We have shown in this Letter that the peak flux is constant even in the fast cooling limit if the evolution is adiabatic. The fast cooling stage has generally not been treated by other authors. We show that the position of the peak of the spectrum varies as $\nu_{c} \propto t^{-1 / 2}$ during fast cooling compared to $\nu_{m} \propto t^{-3 / 2}$ in slow cooling (Fig. 1), and this is one way of distinguishing between the two cases. We have also derived the scalings for a fully radiative evolution, where all the shock energy is radiated efficiently. This regime again has rarely been discussed in the literature. We find that the peak flux decreases with time as $F_{\nu, \max } \propto t^{-3 / 7}$ and the position of the peak varies as $\nu_{c} \propto t^{-2 / 7}$. (These results differ from those given in Katz \& Piran 1997, who considered the flux at $\nu_{m}$ instead of the peak flux, which is at $\nu_{c}$.)

Even within the adiabatic case, we find that there are two possible slopes for the decaying part of the light curve. Writing the flux as $F_{\nu} \sim t^{-\alpha}$, the two cases give $\alpha=3 p / 4-3 / 4$ and $\alpha=3 p / 4-1 / 2$. If the physics of particle acceleration in relativistic shocks is universal in the sense that the power law index $p$ of the electron distribution is always the same, and if the evolution is adiabatic, then we expect always to observe one of these two values of $\alpha$, which differ by $1 / 4$. Indeed, some X-ray afterglows appear to decay with $\alpha \cong 1.4$ while the optical and X-ray afterglows of GRB 970228 and GRB970508 had $\alpha \cong 1.2$ (Yoshida et al. 1997, Sokolov et. al. 1997). The difference between the two values is consistent with $1 / 4$. The corresponding value of $p$ is $\sim 2.5-2.6$, which is a reasonable energy index for shock acceleration. If future observations of $\gamma$-ray burst afterglows always find decays with either $\alpha=1.4$ or $\alpha=1.2$, it will be a strong confirmation of the shock model and the adiabatic assumption. The characteristic values of $\alpha$ are different 
for radiative evolution.

In addition to the decay of the light curve with time, we can also consider the spectral index $\beta$, defined by $F_{\nu} \sim \nu^{-\beta}$. The two values of $\alpha$ given above for adiabatic evolution correspond to $\beta=(p-1) / 2$ and $\beta=p / 2$, respectively. Thus, the relation between $\alpha$ and $\beta$ in an adiabatic fireball is either $\alpha=3 \beta / 2$ or $\alpha=3 \beta / 2+1 / 2$. Previous studies (Rees \& Mészáros 1996, Waxman 1997a) have considered the first possibility. However, we note that, for the standard choice of parameters, namely $n \sim 1 \mathrm{~cm}^{-3}$ (a standard interstellar medium), $\epsilon_{e}, \epsilon_{B}>0.1$ (rough equipartition of energy between electrons and magnetic fields), the second relation holds in both the optical and X-ray bands during much of the decay. Indeed, this relation is more compatible with detailed observations of GRB 970508 (Sokolov et. al. 1997).

Finally, we note that in none of the cases considered does the flux rise more steeply than $t^{1 / 2}$. This is a potential problem since GRB970508 displayed a sharp rise in the optical flux just before its peak at two days (Sokolov et. al. 1997).

This work was supported by NASA Grant NAG5-3516, and a US-Israel BSF Grant 95-328. Re'em Sari thanks The Clor Foundation for support.

\section{REFERENCES}

Blandford, R. D. \& McKee, C. F. 1976, Phys. of Fluids, 19, 1130

Costa, E. et al. 1997a, IAU Circular No. 6572

Frail, D. A., Kulkarni, S. R., Nicastro, L., Feroci, M., \& Taylor, G. B. 1997, Nature, in press.

Goodman, J. 1997, New Astronomy 2(5): 449-460.

Groot, P. J. et al., 1997 IAU Circular No. 6584

Katz \& Piran 1997, ApJ December 1, astro-ph/9706141.

Mészáros, P. \& Rees, M. 1997, ApJ, 476, 232

Panaitescu A. \& Mészáros, P., 1997, submitted to ApJ, astro-ph/9709284

Sari, R. 1997a, ApJL, 489, L37.

Sari, R. 1997b, submitted to ApJL, astro-ph/9709300.

Sari, R., Narayan, R. \& Piran, T. 1996, ApJ, 473, 204.

Sokolov et. al. 1997, astro-ph/9709093.

Vietri, M. 1997, ApJL, 478, L9.

Wijers, R. A. M. P., Rees M. \& Mészáros, P. 1997, MNRAS, 288, L51.

Waxman, E. 1997a, ApJ, 485, L5.

Waxman, E. 1997b, Nature, submitted, astro-ph/9705229

Waxman, E. 1997c, ApJ, submitted, astro-ph/9705229

Yoshida et al., 1997, to appear in proceeding of the 4th Huntsville symposium. 